\title{
Competences of Clinical Psychologists
}

\author{
EACLIPT Task Force On "Competences of Clinical Psychologists"
}

Clinical Psychology in Europe, 2019, Vol. 1(2), Article e35551, https://doi.org/10.32872/cpe.v1i2.35551

Received: 2019-04-18 • Accepted: 2019-06-07 • Published (VoR): 2019-06-28

Handling Editor: Cornelia Weise, Philipps-University of Marburg, Marburg, Germany

Corresponding Author: Winfried Rief, Ph.D., Professor of Clinical Psychology and Psychotherapy, University of Marburg, Marburg, Germany. E-mail: rief@uni-marburg.de

\section{Abstract}

Background: Politicians, societies, stakeholders, health care systems, patients, their relatives, their employers, and the general population need to know what they can expect from clinical psychologists. Even more, for our self-definition as a professional group, we should share a common understanding of the competence profile that characterises our qualifications. This understanding of the competence profile of clinical psychology leads directly to the content that should be taught in university curricula and postgraduate trainings for clinical psychology. The following discussion paper attempts to offer a general European framework for defining the competence profile of clinical psychologists.

Method: A group of European specialists developed this discussion paper under the umbrella of the European Association of Clinical Psychology and Psychological Treatment (EACLIPT). Representatives with different treatment orientations, of basic science and clinical applications, and from East to Western European countries, were part of the group.

Results: We present a list of competences that should be acquired during regular studies of psychology with a clinical specialisation. Additionally, further competences should be acquired either during studying, or during postgraduate trainings.

Conclusion: Clinical psychologists are experts in mental and behavioural disorders, their underlying psychological, social and neurobiological processes, corresponding assessments/ diagnostic tools, and evidence-based psychological treatments. While we provide a list with all competences of clinical psychologists, we do not consider this proposal as a final list of criteria, but rather as a living discussion paper that could be updated regularly. Therefore, we invite our colleagues to contribute to this discussion, and to submit comments via email to the corresponding author.

\section{Keywords}

competences, clinical psychology, psychotherapy, mental disorders 


\section{Highlights}

- People need to know what they can expect from clinical psychologists.

- We present a list of competences that clinical psychologists acquire during their training.

- This list of competences was developed by colleagues representing different treatment orientations, different European countries, and basic versus clinical scientists.

- This competence list can represent a basis for optimising education and training programmes for clinical psychologists, and for informing the public.

Competence lists are increasingly important for the self-definition of a profession, for the planning of study and training curricula, and for the public view on a professional field. Politicians, societies, stakeholders, health care systems, patients, their relatives, their employers, and the general population need to know what they can expect from clinical psychologists. For our self-definition as a professional group, we should share a common understanding of the competence profile that characterises our qualifications. This understanding of the competence profile of clinical psychology leads directly to the content that should be taught in university curricula and postgraduate training for clinical psychology. Therefore, competence lists can be considered as an interactive aspect of the progress of a profession: first, they are developed based on current understanding, reality, experiences, and concepts, but vice versa, the list of competences can be used to develop and improve existing training curricula to better focus on an optimised education of these necessary competences. This interaction is outlined in Figure 1.

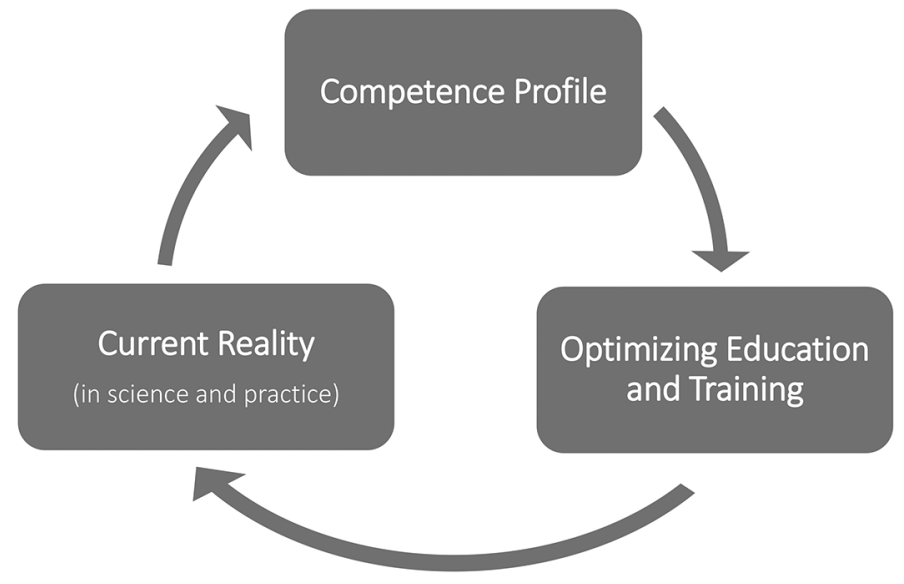

Figure 1. How competence profiles, current practice and education inform each other. 


\section{How Competence Profiles Stimulate the Progress of a Profession}

Many activities exist to develop and improve competence lists for clinical psychologists and psychotherapists. However, many of them are limited to specific nations (Bartolo, 2005) or to specific psychotherapeutic orientations (Sburlati, Schniering, Lyneham, \& Rapee, 2011). In the context of the IAPT programme in UK, the University College of London developed competence frameworks for specific treatment modalities and their supervision (Roth \& Pilling, 2008). They also provided competence profiles for different clinical groups, and clinical contexts (ucl.ac.uk/CORE/). These kinds of competence lists serve to quality assurance, but also to ethical evaluations (Lane, 2011). Some attempts used qualitative methods to approach the field (Nodop \& Strauß, 2014), and differentiated scientific-conceptual competences, personal, and interpersonal competences. Competence lists also play a role in the development of national legal regulations for psychologists and psychotherapists (Willutzki, Fydrich, \& Strauß, 2015).

The aim of this article was to develop and present a European framework of competence profiles for clinical psychologists that should be valid for all evidence-based treatment orientations in all European countries. Therefore, we used the framework of the European Association of Clinical Psychology and Psychological Treatment (EACLIPT) to establish a work group representing different European countries and their national specialties, different treatment orientations, the broad range from basic to applied science, but also further aspects of diversity. The proposal was further evaluated and approved by the EACLIPT board members. Here we present the first version of the European competence list of clinical psychologists.

\section{Competences of Clinical Psychologists}

Clinical psychologists are experts in mental and behavioural disorders, the continuum from mental health to disease, psychological and psychobiological mechanisms of mental and behavioural disorders and physical diseases, epidemiological and health economic relevance of mental and behavioural disorders, vulnerability and resilience factors of psychological health, and evidence-based treatments for mental disorders and psychological factors of physical diseases. Clinical psychologists are engaged in diagnosing, treating and scientifically investigating mental and behavioural disorders and psychological factors of physical diseases within a bio-psycho-social and developmental framework. They plan, conduct, and evaluate activities to promote mental and behavioural health on a scientific basis in prevention, treatment and rehabilitation. They do not only apply current scientific knowledge, but they are also able to work with new complex problems and professional challenges, in a permanently changing environment. They have the competence 
to support the scientifically-driven progress of the field, and to permanently integrate the latest scientific findings into their work.

\section{List of Competences}

\section{More detailed competences of clinical psychologists are:}

a) General Psychological Processes in Health and Disease

Clinical psychologists are experts in identifying and describing psychological, psychosocial, psychobiological and neuroscientific aspects of normal and abnormal human behaviour and experiences, hereby considering the whole life span. They have expertise in analysing the role of cognitive processes such as perception, learning, memory, language, of emotional and motivational processes, in developmental psychology and developmental psychopathology of the whole life span, of the biological basis of human experiences and behaviour, individual differences and dimensions of personality, and they can identify the social and cultural influences on normal and abnormal behaviour and experiences. They are familiar with scientifically sound models to better understand normal and abnormal behaviour, and can apply them to understand and treat psychological problems across life span.

b) Mental and Behavioural Disorders and Psychological Processes in Physical Disorders

Clinical psychologists are experts in informing the public, political stakeholders, institutions, affected people and their relatives about psychological problems and mental disorders, their varying appearances, and how to classify them. They can also identify psychological and psychosocial aspects of physical diseases. They are able to detect, diagnose, classify and describe mental disorders and psychological processes of physical diseases, using observational techniques, self-rating scales, expert ratings and other evaluated assessment tools. Clinical psychologists reflect on cultural, societal and historical relativism in diagnosing mental and behavioural disorders and continually contribute to the development of international classification systems.

c) Psychological Diagnostics

Clinical psychologists are able to develop, evaluate, employ, analyse, and report results of diagnostic tools to improve the objectivity, reliability and validity of diagnosing psychological, psychosocial and neurobiological aspects of mental and behavioural disorders and psychological mechanisms relevant in physical diseases. In their diagnostic work, they consider the continuum between healthy and clinically relevant processes, age- and socioeconomically relevant aspects, and other environmental and cultural determinants of psychological well-being and dysfunctional processes. They employ best-evidence self-rating scales, scientifically evaluated interview techniques, and other assessment tools, both for clinical 
purposes, but also to assess personality characteristics, performance profiles, deficits, skills, and resources.

\section{d) Intervention: General Aspects}

Clinical psychologists know about the different evidence-based psychological interventions, their historical development and current evidence-based evaluation. They can critically think about different treatments and can inform the public about scientifically based treatment guidelines and typical treatment characteristics, hereby considering disorder-, person- and sociocultural-relevant aspect. They use scientifically-based interventions to enhance resources of the patients and clients to improve psychological well-being, and to reduce vulnerability and risk factors for psychological problems and mental and behavioural disorders. Clinical psychologists can inform patients, their relatives, public institutions, stakeholders and others about the potential and risks of psychological treatments, based on a current critical scientific evaluation of them.

\section{e) Prevention, Rehabilitation: General Academic Expertise}

Clinical psychologists are able to inform about prevention and rehabilitation programmes, their scientific evidence, and their potential use for society and specific target groups. They can develop, apply and evaluate such programmes. Clinical psychologists can promote mental and behavioural health and develop mental health literacy in various settings.

\section{f) Scientific Methodology}

Clinical psychologists can use qualitative and quantitative approaches to investigate psychological, psychobiological and psychosocial processes and clinical applications to better understand normal and abnormal behaviour and experiences. They are able to plan, conduct and analyse the results of studies using modern criteria for scientific evaluations and advanced statistical modelling. In particular, clinical psychologists are able to plan, conduct, analyse, report and explain clinical trials and their results, to evaluate psychological interventions according to modern scientific standards. They are also able to understand and use methods and results from developmental, cognitive and experimental psychology, or from any other field related to scientific psychology important for the understanding of the aetiology, maintenance and treatment of mental and behavioural disorders. They know methods and central elements of psychotherapy and psychological intervention research, and how to incorporate that knowledge into their clinical practice. They actively take part in psychological intervention and psychotherapy research by developing research questions, designs and treating patients in clinical trials.

\section{g) Ethical and Legal Aspects}

Clinical psychologists consider and respect current ethical standards and legal regulations for their professional work. Clinical psychologists are sensible regarding cultural diversity and respect it in their work with clients. 
Depending on national regulations, the following skills are either acquired during university studies, or during postgraduate trainings often connected to the term psychotherapy:

\section{h) Skills for Psychological Interventions: Meta-Competences}

- Clinical psychologists are able to provide psychological interventions that follow treatment aims and a treatment plan, based on current scientific knowledge about mental and behavioural disorders and interventions. Major competences to provide interventions have been acquired according to current standards of learning how to practise these interventions.

- Clinical psychologists are able to motivate patients to engage in psychological interventions, and to foster and maintain a good alliance with their clients/ patients, their relatives and significant others. They can explain the intervention rationale to patients, other health care specialists, and further involved people.

- Clinical psychologists have the competence for perspective taking, empathy and mentalisation. They have professional skills to identify the diversity of verbal and nonverbal communication signals of others. They have professional competences to communicate with others, based on a broad variety of acquired communication skills, even during difficult communication sequences, or with patients with difficult communication patterns. They can verbally address emotional, cognitive, behavioural and interactive aspects of the patient's/client's behaviour.

- Clinical psychologists have an advanced ability to regulate their own emotions and behaviour, and to reflect their own emotions, cognitions, and behaviour during professional encounters. They can reflect the consequences of past learning and socialisation processes on current behaviour and experiences, not only in others, but also in themselves. They can cope with professional stressful situations, but are able to relax and plan their life according to an adequate work-life-balance.

- Clinical psychologists are able to evaluate on-going interventions of themselves or of others, to detect unfavourable or unexpected events, and to react adequately in the event of occurring risks (e.g., suicidality). They are able to address treatment problems (e.g. adherence problems of patients) and problems of the therapeutic relationship accordingly. They are able to use the patient's/client's feedback to adapt intervention processes.

- When confronted with new problematic professional situations, they have concepts about how to develop new problem-solving strategies, based on a profound framework theory how to plan interventions.

- Clinical psychologists are able to end interventions in a planned manner, to plan for long-term maintenance of treatment gains, and to reduce the risk of relapse after the intervention. 
- Clinical psychologists aim to continuously improve their professional abilities. They are able to learn from their own and other experiences, from supervision and intervision, and to transfer current scientific knowledge to clinical practice and to integrate recommendations of others (e.g. supervisors) in their clinical work.

- Clinical psychologists are able to communicate with other health-professionals, and to coordinate their diagnostic and intervention plans with other experts involved in the overall treatment plan.

i) Skills for Psychological Interventions: Disorder-, Person-, and ContextSpecific Diagnostics and Interventions

Following recommendations of official scientifically based guidelines, clinical psychologists can select evidence-based diagnostic tools and evidence-based psychological interventions for specific mental and behavioural disorders and psychological aspects of physical diseases. For treatment planning, they consider the different severity degrees and courses of mental and behavioural disorders, the comorbidity profiles, further associated problems, the patient's and setting's resources, as well as cultural aspects.

j) Skills for Psychological Interventions: Prevention, Rehabilitation

Clinical psychologists are able to prepare, conduct, and evaluate clinical prevention and rehabilitation programmes according to current scientific standards. They have public relation skills to present programmes and persuasive skills to promote the relevance of the programmes to significant stakeholders.

k) Skills for Psychological Interventions: Setting-Specific Interventions, Modern Technologies

Clinical psychologists are able to provide professional, scientifically based work with individuals, with couples, with families, and in groups. They can provide expert knowledge and they have the ability to work in complex systems (e.g., hospitals, occupational health services, political institutions). They are aware of options to increase effectivity, reachability, and benefit-cost-ratios for providing clinical psychological interventions also by using modern technologies.

1) Skills for Psychological Interventions: Documentation, Evaluation Clinical psychologists continuously document and evaluate their work. They consider aspects of quality insurance.

\section{Conclusion}

We provide a first list with the characterising competences of clinical psychologists that aims to cover the needs of all European nations, but also of representatives of different specialisations and orientations of clinical psychology. However, we do not consider this proposal as an exhaustive list of criteria, but as a living discussion paper that could be 
updated regularly. Therefore, we invite our colleagues to contribute to this discussion, and to submit comments via email to the corresponding author.

Funding: The authors have no funding to report.

Competing Interests: The authors have declared that no competing interests exist.

Acknowledgments: The authors have no support to report.

Author Note: Members of the EACLIPT task force on "Competences of Clinical Psychologists" and the EACLIPT board which has approved this proposal are: Gerhard Andersson, Natasa Jokic Begic, Claudi Bockting, Roman Cieslak, Celine Douilliez, Thomas Ehring, Philipp Kanske, Andreas Maercker, Agnieszka Popiel, Winfried Rief, Chantal Martin Soelch, Svenja Taubner.

\section{References}

Bartolo, P. A. (2005). Regulating the psychology profession in Malta. European Psychologist, 10(1), 76-77. https://doi.org/10.1027/1016-9040.10.1.76

Lane, D. (2011). Ethics and professional standards in supervision. In T. Bachkirova, P. Jackson, \& D. Clutternuck (Eds.), Coaching and mentoring supervision: Theory and practice (pp. 99-104). Maidenhead, United Kingdom: Open University Press.

Nodop, S., \& Strauß, B. (2014). Kompetenzbereiche in der psychotherapeutischen Ausbildung. Zeitschrift für Klinische Psychologie und Psychotherapie, 43(3), 171-179. https://doi.org/10.1026/1616-3443/a000272

Roth, A. D., \& Pilling, S. (2008). A competence framework for the supervision of psychological therapies. Retrieved August, 18, 2011 from www.ucl.ac.uk/CORE/.

Sburlati, E. S., Schniering, C. A., Lyneham, H. J., \& Rapee, R. M. (2011). A model of therapist competencies for the empirically supported cognitive behavioral treatment of child and adolescent anxiety and depressive disorders. Clinical Child and Family Psychology Review, 14(1), 89-109. https://doi.org/10.1007/s10567-011-0083-6

Willutzki, U., Fydrich, T., \& Strauß, B. (2015). Aktuelle Entwicklungen in der Psychotherapieausbildung und der Ausbildungsforschung. Psychotherapeut, 60(5), 353-364. https://doi.org/10.1007/s00278-015-0048-1 


\section{EACLIPT}

Clinical Psychology in Europe (CPE) is the official journal of the European Association of Clinical Psychology and Psychological Treatment (EACLIPT).

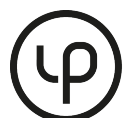

leibniz-psychology.org

PsychOpen GOLD is a publishing service by Leibniz Institute for Psychology Information (ZPID), Germany. 